\title{
New evidences on the altered gut microbiota in autism spectrum disorders
}

\author{
Francesco Strati ${ }^{1,2}$, Duccio Cavalieri ${ }^{3}$, Davide Albanese ${ }^{1}$, Claudio De Felice ${ }^{4}$, Claudio Donati ${ }^{1}$, Joussef Hayek ${ }^{5,6}$, \\ Olivier Jousson ${ }^{2}$, Silvia Leoncini ${ }^{5}$, Daniela Renzi ${ }^{7}$, Antonio Calabrò ${ }^{7}$ and Carlotta De Filippo ${ }^{{ }^{*}}$
}

\begin{abstract}
Background: Autism spectrum disorders (ASDs) are neurodevelopmental conditions characterized by social and behavioural impairments. In addition to neurological symptoms, ASD subjects frequently suffer from gastrointestinal abnormalities, thus implying a role of the gut microbiota in ASD gastrointestinal pathophysiology.

Results: Here, we characterized the bacterial and fungal gut microbiota in a cohort of autistic individuals demonstrating the presence of an altered microbial community structure. A fraction of $90 \%$ of the autistic subjects were classified as severe ASDs. We found a significant increase in the Firmicutes/Bacteroidetes ratio in autistic subjects due to a reduction of the Bacteroidetes relative abundance. At the genus level, we observed a decrease in the relative abundance of Alistipes, Bilophila, Dialister, Parabacteroides, and Veillonella in the ASD cohort, while Collinsella, Corynebacterium, Dorea, and Lactobacillus were significantly increased. Constipation has been then associated with different bacterial patterns in autistic and neurotypical subjects, with constipated autistic individuals characterized by high levels of bacterial taxa belonging to Escherichia/Shigella and Clostridium cluster XVIII. We also observed that the relative abundance of the fungal genus Candida was more than double in the autistic than neurotypical subjects, yet due to a larger dispersion of values, this difference was only partially significant.
\end{abstract}

Conclusions: The finding that, besides the bacterial gut microbiota, also the gut mycobiota contributes to the alteration of the intestinal microbial community structure in ASDs opens the possibility for new potential intervention strategies aimed at the relief of gastrointestinal symptoms in ASDs.

Keywords: Autism spectrum disorders, Gut microbiota, Mycobiota, Constipation, Metataxonomy

\section{Background}

The term "autism spectrum disorders" (ASDs) refers to a group of neurodevelopmental disorders with an early life stage onset characterized by alterations in social interactions and communication and by restricted and repetitive behaviour [1]. It is now well accepted the contribution of both genetic and environmental factors in the aetiology of ASDs [2,3]. Among the non-neurological symptoms associated with ASDs, several studies indicate gastrointestinal (GI) symptoms as common comorbidities [4-7]. Alterations in the composition of the gut microbiota have been implicated in a wide variety of human diseases, including ASDs [8]. Since the gut microbiota makes critical contributions to metabolism and maintenance of

\footnotetext{
* Correspondence: carlotta.defilippo@ibba.cnr.it

${ }^{8}$ Institute of Agriculture Biology and Biotechnology, National Research

Council (CNR), Via Moruzzi 1, 56124 Pisa, Italy

Full list of author information is available at the end of the article
}

immune homeostasis and may control the central nervous system (CNS) activities through neural, endocrine, and immune pathways [9], it has been hypothesized the active role of the gut microbiota in ASD pathophysiology. There is more than a subtle link between the gut microbiota and the CNS, through the so-called "microbiome-gut-brain axis". Indeed, it has been demonstrated a direct interaction between the gut microbiota and enteric neurons $[10,11]$, its role in the regulation of the HPA axis [12], and the production of many chemicals important in brain functioning (e.g., serotonin, dopamine, kynurenine, $\gamma$-aminobutyric acid, SCFAs, $p$-cresol) $[13,14]$. A dysbiotic microbial community could lead to systemic inflammation due to hyper-activation of T-helper 1 and T-helper 17 cell responses [15] affecting also the reactivity of peripheral immune cells to the CNS [16] and the integrity of blood-brain barrier [17] which is known to be altered in ASDs [18]. Several evidences suggested 
an early immune activation with chronic inflammation and cytokine dysregulation in ASDs $[19,20]$, and it has been shown that systemic inflammation induced by LPS provokes behavioural changes and impairs the blood-brain barrier in animal models [17, 21]. Furthermore, fungal infections that may originate from alterations in commensal bacteria population [22] could shift the indoleamine 2,3-dioxygenase's activity [23, 24] reducing the levels of kynurenine [25], a neuroprotective agent. Despite several reports disclosed an aberrant gut microbiota in ASDs, consensus across studies has not yet been established [8]. Here, we characterized the bacterial gut microbiota and the less studied gut mycobiota of subjects affected by autism through ampliconbased metataxonomic analysis of the V3-V5 regions of the prokaryotic $16 \mathrm{~S}$ ribosomal DNA and of the internal transcribed spacer 1 (ITS1) region of the fungal rDNA in order to better understand the microbial community structure associated with ASDs and its involvement on GI abnormalities.

\section{Results}

Autistic subjects harbour an altered bacterial gut microbiota For the characterization of the gut microbiota associated with autism, we recruited 40 autistic subjects (36 out of 40 autistic subjects were classified as severe ASDs, Childhood Autism Rating Scale (CARS) value $>37$ ) and 40 neurotypical controls (Table 1, Additional file 1: Table S1). Analysis of alpha diversity revealed no significant differences between autistic and neurotypical subjects (hereinafter termed $\mathrm{AD}$ and NT, respectively). However, the analysis of the beta diversity calculated on the unweighted and weighted UniFrac distances and the Bray-Curtis dissimilarity revealed that the bacterial microbiota of $\mathrm{AD}$ clusters apart from that of NT $(p<0.05$, permutational multivariate analysis of variance (PERMANOVA); Fig. 1, Additional file 2: Table S2). Since we enrolled subjects suffering from constipation among NT and AD subjects, the impact of constipation on the beta diversity of the two groups of study was also analysed. We observed that constipation has a significant effect on the microbial community structure within NT subjects $(p<0.05$, PERMANOVA), as expected [26, 27], but not within AD subjects (Additional file 2: Table S2). Furthermore, we observed that the severity of the autistic phenotype, as measured by CARS scores, does not affect the bacterial community structure among $\mathrm{AD}$ individuals $(p>0.05$, PERMANOVA; Additional file 3: Table S3).

Phylum level analysis showed a clear alteration of the bacterial gut community in $\mathrm{AD}$ characterized by a higher Firmicutes/Bacteroidetes ratio $(p<0.005$, Wilcoxon ranksum test; Fig. 2a) in AD than that in NT due to a significant reduction of the relative abundance of Bacteroidetes (9.2\% AD, 19.4\% NT) (FDR-corrected $p<0.05$, Welch $t$
Table 1 Characteristics of study participants

\begin{tabular}{|c|c|c|}
\hline & Autistic & Neurotypical \\
\hline Subjects $(n)$ & 40 & 40 \\
\hline Age (1st-3rd quartile) & $10(5-17)$ & $7(3.6-12)$ \\
\hline \multicolumn{3}{|l|}{ Gender (n) } \\
\hline Female & $22.5 \%(9)$ & $30 \%(12)$ \\
\hline Male & $77.5 \%(31)$ & $70 \%(28)$ \\
\hline \multicolumn{3}{|l|}{ Constipation (n) } \\
\hline Constipated & $12.5 \%(5)$ & $27.5 \%(11)$ \\
\hline Non-constipated & $72.5 \%(29)$ & $72.5 \%(29)$ \\
\hline NA & $15 \%(6)$ & $0 \%(0)$ \\
\hline $\begin{array}{l}\text { Calprotectin } \\
\text { (1st-3rd quartile) }\end{array}$ & $36.9(17.6-76.0) \mu \mathrm{g} / \mathrm{g}$ & $40.9(17-74.7) \mu \mathrm{g} / \mathrm{g}$ \\
\hline Constipated & $39.1(22.9-70.0) \mu \mathrm{g} / \mathrm{g}$ & $27.9(20.3-97.6) \mu \mathrm{g} / \mathrm{g}$ \\
\hline Non-constipated & $35.9(15.0-57.8) \mu \mathrm{g} / \mathrm{g}$ & $50.5(15.0-73.8) \mu \mathrm{g} / \mathrm{g}$ \\
\hline CARS (1st-3rd quartile) & $47(40-50.5)$ & NA \\
\hline Constipated & $50(36-52.0)$ & NA \\
\hline Non-constipated & $48(42-50.0)$ & NA \\
\hline ESR (1st-3rd quartile) & $7.5(3.25-17.7) \mathrm{mm} / \mathrm{h}$ & NA \\
\hline Constipated & $22.0(12.0-25.0) \mathrm{mm} / \mathrm{h}$ & NA \\
\hline Non-constipated & $7.0(2.7-11.2) \mathrm{mm} / \mathrm{h}$ & NA \\
\hline $\begin{array}{l}\text { Serum IgA } \\
\text { (1st-3rd quartile) }\end{array}$ & $131.0(70.0-172.2) \mathrm{mg} / \mathrm{ml}$ & NA \\
\hline Constipated & $97.0(82.0-153.0) \mathrm{mg} / \mathrm{ml}$ & NA \\
\hline Non-constipated & $133.0(67.0-181.0) \mathrm{mg} / \mathrm{ml}$ & NA \\
\hline
\end{tabular}

Data expressed as medians with interquartile ranges when applicable. $A D$ autistic subjects, NT neurotypical subjects, NA not applicable, CARS Childhood Autism Rating Scale, ESR erythrocyte sedimentation rate

test; Fig. 2b). Genus level analysis showed that the top ten most abundant genera in both AD and NT subjects were Bifidobacterium, Bacteroides, Faecalibacterium, Unknown Lachnospiraceae, Blautia, Ruminococcus, Clostridium XI, Streptococcus, Gemmiger, and Lachnospiraceae incertae sedis (Additional file 4: Figure S1, Additional file 5: Table S4). Interestingly, the genus Prevotella was only barely represented in AD with respect to NT $(0.05 \%$ $\mathrm{AD}, 1.5 \% \mathrm{NT}$ ), in agreement with a previous study on the gut microbiota in ASD children [28] although this difference of relative abundance was not supported by the statistical analysis. We further analysed the bacterial community structure associated with AD and NT by using linear discriminant effect size (LEfSe), an algorithm for high-dimensional biomarker discovery which uses linear discriminant analysis (LDA) to estimate the effect size of each taxon which is differentially represented in cases and controls [29]. LEfSe analysis revealed a significant increase of the relative abundance of different bacterial taxa in $\mathrm{AD}$ than in NT among which Collinsella, Corynebacterium, Dorea, and Lactobacillus and a significant reduction of the taxa Alistipes, Bilophila, Dialister, Parabacteroides, 

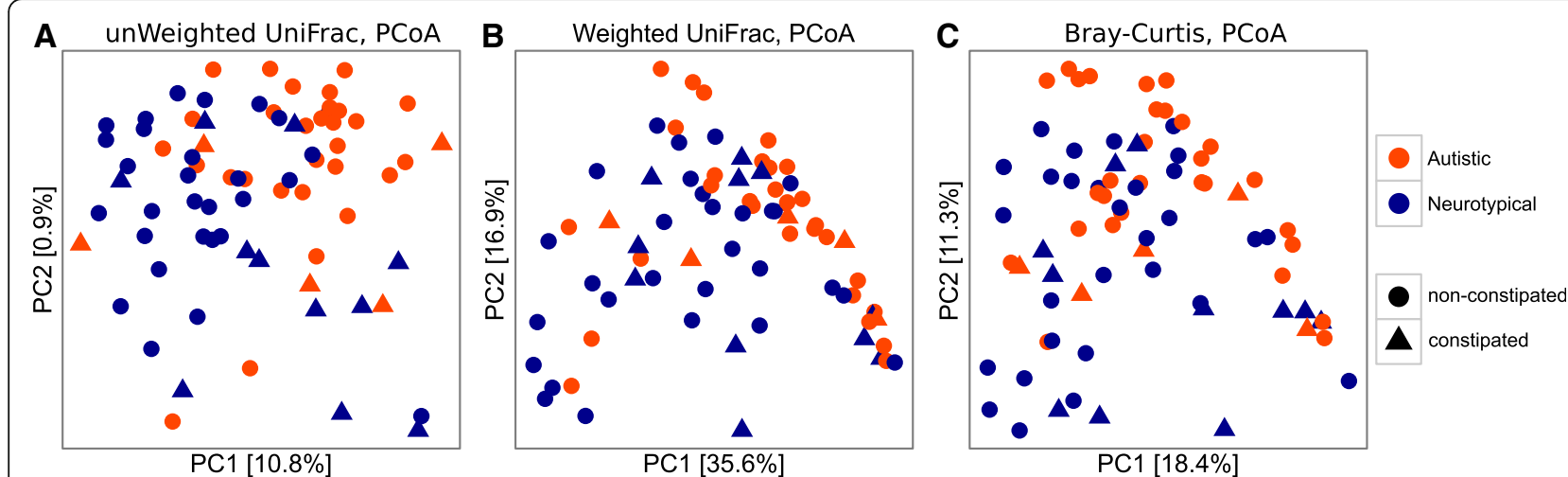

Fig. 1 PCoA of bacterial beta diversity based on the $\mathbf{a}$ unweighted and $\mathbf{b}$ weighted UniFrac distances and $\mathbf{c}$ Bray-Curtis dissimilarity. Autistic and neurotypical subjects are coloured in orange and blue, respectively. The constipation status of the subjects is indicated according to different shapes, circles for non-constipated and triangles for constipated individuals

and Veillonella in AD than in NT $(p<0.01$, Wilcoxon rank-sum test; LDA >2.0; Fig. 3).

\section{Constipation selects different bacterial taxa in autistic subjects and neurotypical healthy controls}

Autistic subjects frequently suffer of GI comorbidities [4-7], and constipation is a GI symptom often reported in these subjects, known to alter the physiology of the human GI tract and the gut microbiota itself [27, 30, 31]. Correlation analysis of the bacterial relative abundances between constipated and non-constipated subjects, both autistic and neurotypical, revealed that among the most abundant bacterial genera (with relative abundance $>0.5 \%$ and detectable in at least the $70 \%$ of the investigated subjects), the taxa Gemmiger and Ruminococcus anticorrelates with the constipation status (Spearman's correlation $r=-0.39$ and -0.36 , respectively; FDR-corrected $p<0.05$; Additional file 6: Table S5) while Escherichia/Shigella and Clostridium cluster XVIII positively correlates with this GI symptom (Spearman's correlation $r=0.31$ and 0.38 , respectively; FDR-corrected $p<0.05$; Additional file 6 : Table S5). We further compared the relative abundance of these taxa among constipated and non-constipated subjects within and between groups. We observed that Escherichia/Shigella and Clostridium cluster XVIII were significantly more abundant in constipated AD compared to the non-constipated ones (FDR-corrected $p<0.05$, Wilcoxon rank-sum test; Fig. 4a, b) while no differences have been detected between constipated and nonconstipated NT for these taxa. On the other hand, the genus Gemmiger was significantly less abundant in constipated compared to non-constipated NT (FDR-corrected $p<0.05$, Wilcoxon rank-sum test; Fig. 4c). Remarkably, no significant differences have been observed in the levels of faecal calprotectin between AD and NT as well as between constipated and non-constipated subjects in both groups (Table 1 and Additional file 1: Table S1). Furthermore, we analysed the levels of other two biomarkers of inflammations, i.e., serum IgA and ESR in the autistic subjects, and we did not observe significant differences among constipated and non-constipated AD (Table 1 and Additional file 1: Table S1). Therefore, while constipation resulted in a significant increase of Escherichia/ Shigella and Clostridium cluster XVIII, no differences have
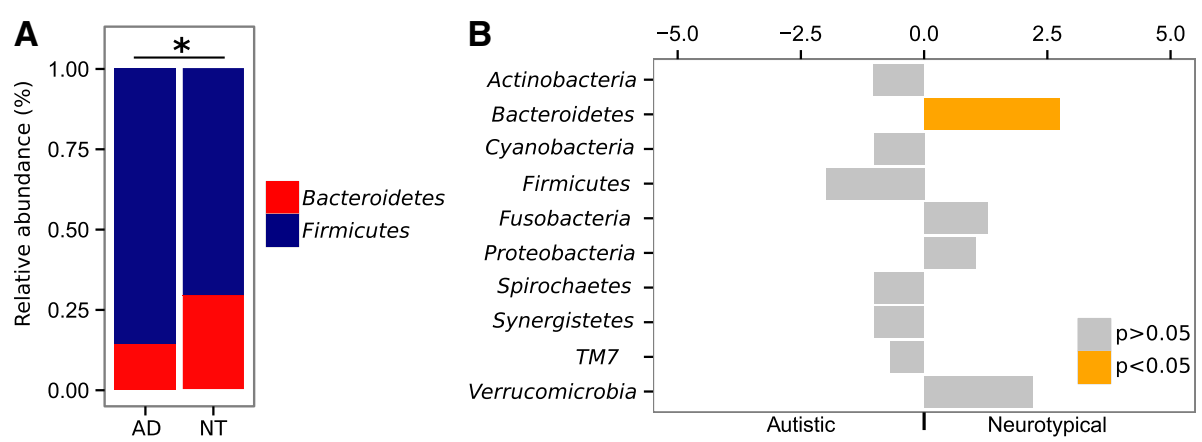

Fig. 2 a Mean relative abundances (\%) of Firmicutes and Bacteroidetes in autistic (AD) and neurotypical (NT) subjects; ${ }^{*} p<0.005$, Wilcoxon rank-sum test on the Firmicutes/Bacteroidetes ratio. $\mathbf{b}$ Welch's $t$ test statistics of the relative abundances of bacterial phyla in autistic and neurotypical subjects. Orange bars indicate significant FDR-corrected $p$ values adjusted for multiple comparison controlling the family-wise type I error rate 


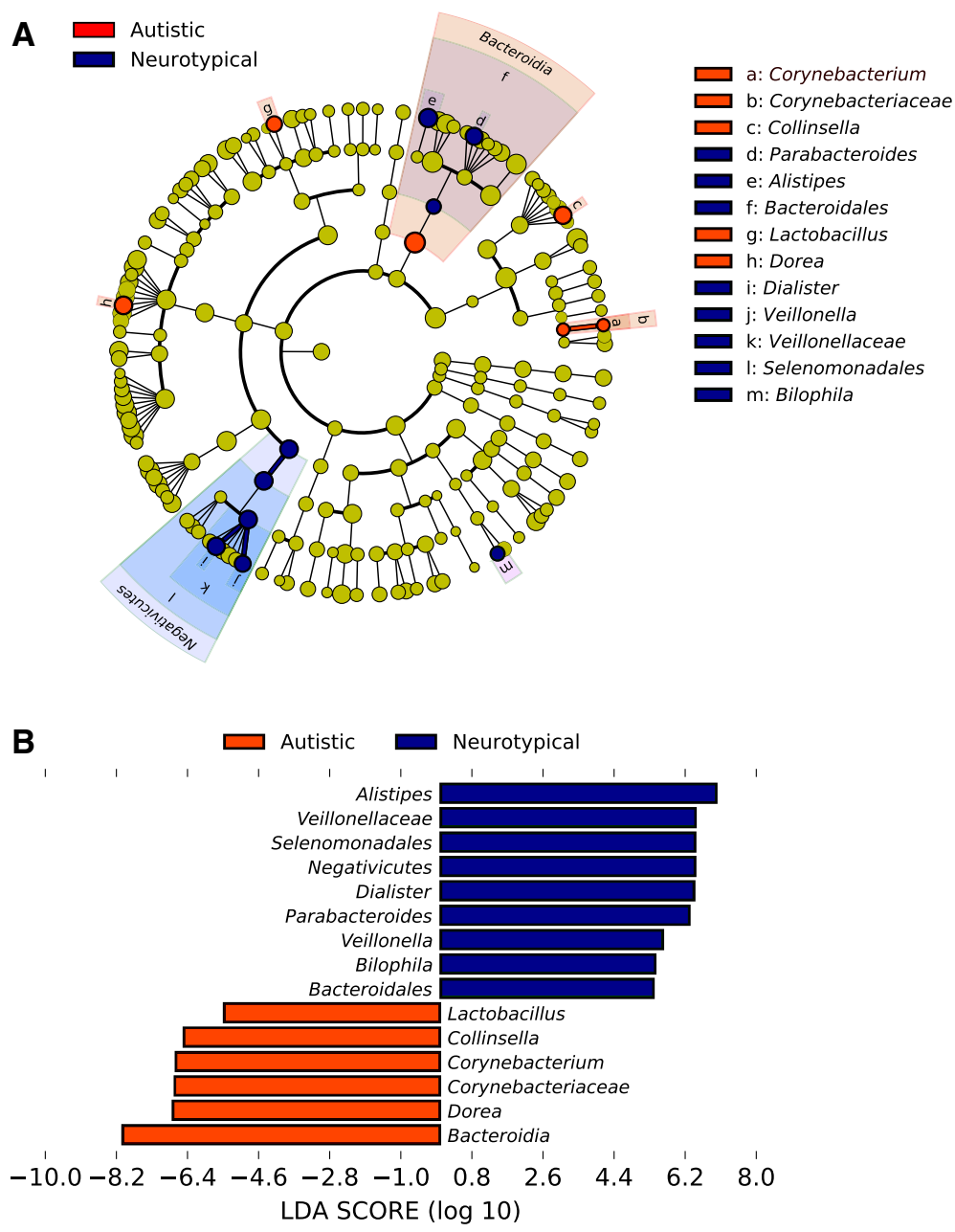

Fig. 3 a Cladograms generated by LEfSe indicating differences in the bacterial taxa between autistic (AD) and neurotypical (NT) subjects. Nodes in orange indicate taxa that were enriched in AD compared to those in NT, while nodes in blue indicate taxa that were enriched in NT compared to those in AD. $\mathbf{b}$ LDA scores for the bacterial taxa differentially abundant between autistic (AD) and neurotypical (NT) subjects. Positive and negative LDA scores indicate the bacterial taxa enriched in NT and AD subjects, respectively. Only the taxa having a $p<0.01$ (Wilcoxon rank-sum test) and LDA $>2.0$ are shown in the figure legend

been observed in the levels of inflammation between constipated and non-constipated autistic subjects suggesting that constipation and the related alterations of the gut microbiota in autistic subjects as well as in neurotypical individuals are not associated with an increase of intestinal inflammation. It should be noted that the number of enrolled constipated subjects was quite low and therefore these analyses could be underpowered.

\section{Autistic subjects harbour an altered gut mycobiota}

We then investigated the gut mycobiota of our study cohort through amplicon-based sequencing of fungal ITS1 region. High-quality fungal sequences were detected respectively in 35 out of 40 autistic subjects and 38 out of 40 NT. As occurred for the bacterial gut microbiota, we did not observe significant differences in fungal alpha diversity between AD and NT. The analysis of beta diversity revealed that the gut mycobiota of $\mathrm{AD}$ was different compared to NT as calculated by principal coordinates analysis (PCoA) and PERMANOVA on the weighted UniFrac distance and Bray-Curtis dissimilarity $(p<0.05$; Fig. 5). As for the bacterial beta diversity, constipation showed a significant effect within NT subjects ( $p=0.046$, PERMANOVA on Bray-Curtis dissimilarities) but not within AD subjects (Additional file 7: Table S6). Furthermore, the severity of the autistic phenotype does not affect the gut mycobiota community structure among $\mathrm{AD}$ individuals $(p>0.05$, PERMANOVA; Additional file 3: Table S3). An in-depth analysis of the gut mycobiota leads to the identification of 50 fungal taxa fully classified to the genus level and 30 only partially classified. Genus level analysis showed 


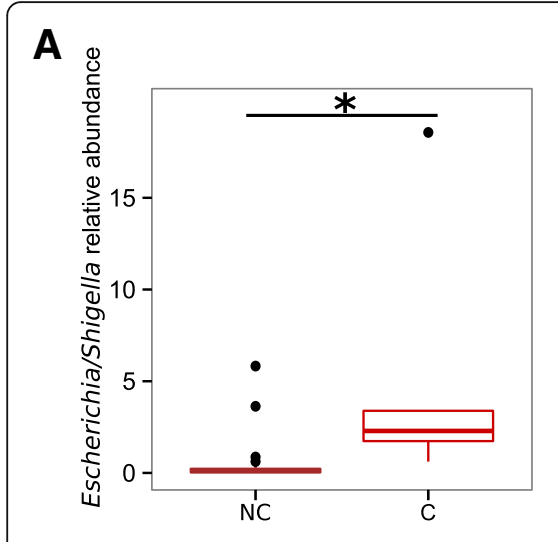

\section{Autistic}

Fig. 4 Box plot representation of the relative abundances of bacterial genera correlating with the constipation status of the subjects enrolled in this study. Comparisons between $\mathbf{a}$ and $\mathbf{b}$ constipated (C) and non-constipated (NC) autistic subjects and (c) constipated (C) and non-constipated (NC) neurotypical subjects; Asterisk indicates FDR-corrected $p<0.05$, Wilcoxon rank-sum test
Aspergillus (24.2\% AD; 28\% NT), Candida (37.7\% AD; $14.1 \%$ NT), Penicillium (13.2\% AD; 23.5\% NT) and Malassezia (3.05\% AD; 3.3\% NT) as the most abundant and widely distributed genera in our study cohort in terms of relative abundance (Additional file 8: Figure S2, Additional file 9: Table S7). The relative abundance of the genus Candida was more than twice as much in $\mathrm{AD}$ than NT, yet due to a large dispersion of values $(p<0.001$; Levene's test), this difference was only partially significant (Welch $t$ test, FDR-corrected $p$ value $=0.09$, uncorrected $p$ value $=0.006$; Additional file 10: Figure S3). The superimposition of the most abundant genera over the PCoA

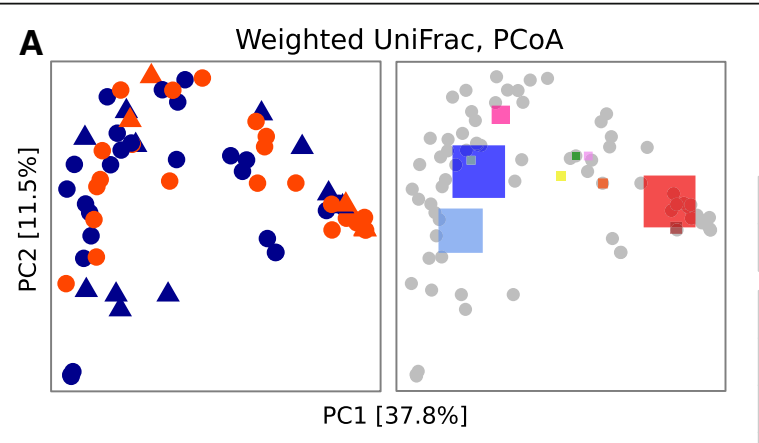

$B$

Bray-Curtis, PCOA
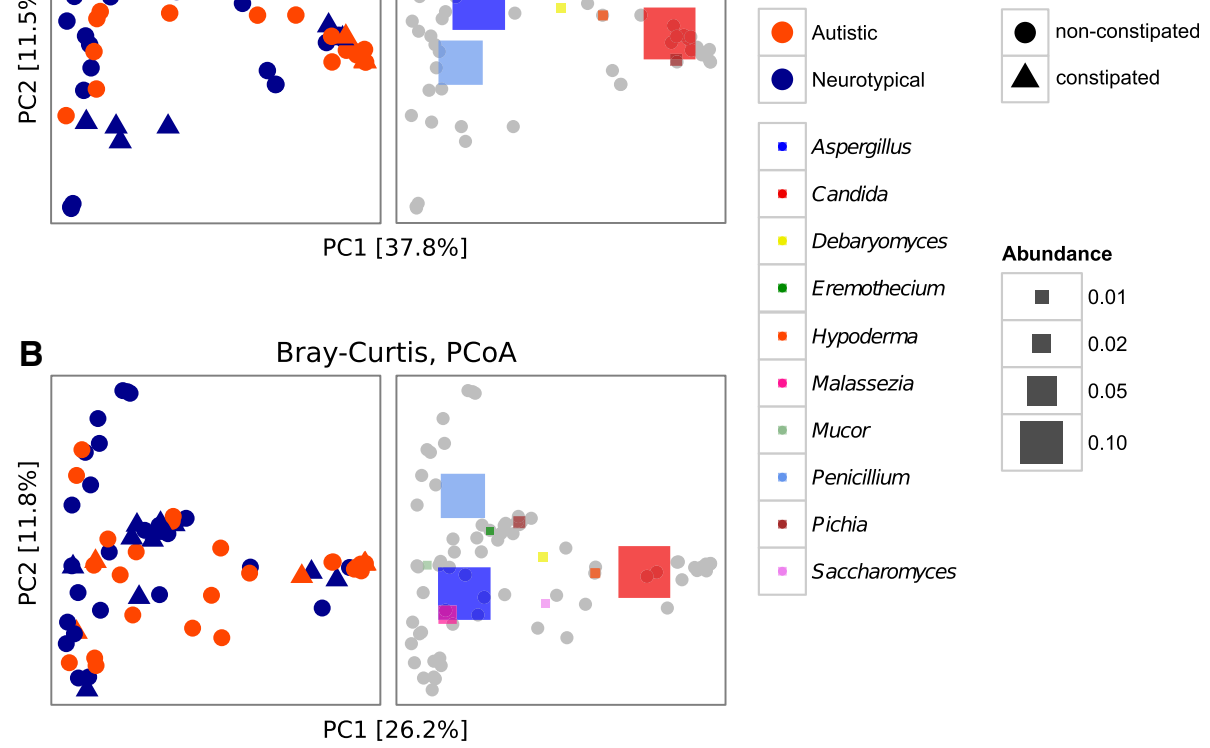

Fig. 5 PCoAs of fungal beta diversity based on a weighted UniFrac distance and $\mathbf{b}$ Bray-Curtis dissimilarity. The right panel of the graphs $\mathbf{a}$ and $\mathbf{b}$ shows the same PCOA coordinates with the most abundant OTUs superimposed as coloured squares, with the size being proportional to the mean relative abundance of the taxon across all samples (grey dots). Autistic and neurotypical subjects are colored in orange and blue, respectively. The constipation status of the subjects is indicated according to different shapes, circles for non-constipated and triangles for constipated individuals 
plots revealed that high levels of Candida abundance was associated with a group of subjects mainly affected by autism (Fig. 5) suggesting that Candida indeed could play a role in the altered microbial community associated with the autistic subjects. Correlation analyses among the most abundant fungi and bacteria (with relative abundance $>0.5 \%$ and detectable in at least the $70 \%$ of the investigated subjects) revealed no significant correlations among autistic subjects while a significant positive correlation between the genera Aspergillus and Bifidobacterium was found within neurotypical individuals (Spearman's $r=0.6$, FDR-corrected $p=0.004$ ) (Additional file 11: Table S8).

\section{Discussion}

The gut microbiota is a crucial factor for the maintenance of the GI tract functions and immune homeostasis. It is well known that dysbiosis of the GI tract could lead to inflammation and immune activation in several pathologies [15]. The frequent occurrence of GI symptoms in autistic subjects imply the possible involvement of the gut microbiota in ASD gastrointestinal pathophysiology, further supported by the speculations on the increased incidence of ASD cases due to "Western" habits (i.e., diet, medications, and excessive overall hygiene) that can affect the composition of the gut microbiota [32]. Several studies demonstrated alterations in the bacterial gut microbiota of ASD individuals, even if the differences reported in these studies were in some cases discordant, possibly due to variance in sampling strategies and methodologies used [8]. In addition, our recent findings showed an altered gut microbiota in Rett syndrome [33], a genetically determined neurodevelopmental disorder previously categorized in the ASD group sharing some features of these conditions. We characterized the gut microbiota associated with autism, disclosing an altered microbial community both at bacterial and fungal level. We observed a significant increase in the Firmicutes/Bacteroidetes ratio in autistic subjects due to a significant reduction of Bacteroidetes in these individuals. Several inflammatory conditions have been related to an increase in the Firmicutes/Bacteroidetes ratio such as inflammatory bowel diseases (IBDs) [34] and obesity [35]. Consistently with these observations, an increased Firmicutes/Bacteroidetes ratio has been reported also in subjects with autism [36, 37]. Furthermore, we discovered that the relative abundances of the genera Collinsella, Corynebacterium, Dorea, and Lactobacillus were significantly increased in the gut microbiota of autistic subjects with respect to that of the neurotypical subjects while the relative abundance of the genera Alistipes, Bilophila, Dialister, Parabacteroides, and Veillonella were significantly reduced in these individuals. A recent study on a mouse model of ASDs demonstrated that treatments with a $\mathrm{PSA}^{+}$Bacteroides fragilis strain restore autism-related behavioural and GI abnormalities, also reducing the reported high levels of Lachnospiraceae and 4-ethylphenyl sulfate, a metabolite produced by this bacterial family related to $p$-cresol, a putative metabolic marker for autism [38]. Overall, these data are consistent with our findings and remark the importance of Bacteroidetes in ASD pathophysiology. Moreover, Lactobacillus resulted to be enriched in the gut microbiota of autistic individuals while Dialister and Veillonella resulted to be depleted, in line with the results obtained in previous studies $[28,39]$. Since constipation is a common gastrointestinal problem in subjects with ASDs [4-7], we compared our data between constipated and non-constipated subjects in order to evaluate the contribution of constipation in shaping the gut microbiota of autistic subjects. Indeed, it has been proposed that GI symptoms may be related to ASDs [40]. The evidence that the taxa belonging to the Clostridium cluster XVIII and the putative proinflammatory Escherichia/Shigella [41, 42] positively correlated with the constipation status of the subjects as well as their enrichment in constipated autistic subjects supports the hypothesis that GI problems and related alterations of the gut microbiota may contribute to ASD gastrointestinal symptoms [40]. Because of their ability to produce exotoxins and propionate that may exacerbate autistic symptoms [43], the role of clostridia in ASDs has been extensively explored. The species belonging to the Clostridium cluster XVIII have been shown to produce exotoxins [44] and to promote conditions favouring inflammation $[45,46]$ although other studies observed their potential ability to induce homeostatic T-reg responses [47]. It is also interesting to underline the occurrence of a subclinical acute phase response in ASD plasma, as evidenced by advanced proteomic analysis [48].

Despite the importance given to the implications of the gut microbiota in health and disease, few reports have explored the relevance of the fungal component of the gut microbiota in GI (patho) physiology [49]. Furthermore, none of the published studies on ASDs' gut microbiota have assessed the fungal gut community structure associated with autism. Our dataset of autistic subjects displayed a different fungal community structure compared to neurotypical subjects. In particular, the genus Candida was one of the most abundant taxa in the gut mycobiota of this study cohort, being two times more abundant in AD than in NT. To the best of our knowledge, this is the first time that alterations of the intestinal fungal microbiota are associated with ASDs. Although Candida is one of the most common and abundant genus of the human gut mycobiota [50,51], its implication in phenomena of fungal dysbiosis have been reported in several GI and inflammatory conditions [52-54] as well as in Rett syndrome [33]. It is therefore possible that 
alteration of the intestinal fungal population driven by an expansion of Candida in the gut mycobiota of autistic individuals may negatively impact on GI abnormalities through cytokine dysregulation. The gut microbiota, in particular some species of Lactobacillus, modulates the immunological responses to Candida in the GI tract by providing tryptophan-derived aryl hydrocarbon receptor ligands that stimulate the immune system, principally ILC3 cells, to produce IL-22 [55]. Together with IL-17, IL22 avoids the excessive proliferation of Candida and other fungal commensals in the gut. It is therefore possible that alterations of the gut microbiota in ASDs could lead to an expansion of the Candida population preventing from full restoration of the bacterial community structure. Indeed, it has been observed that alterations of the bacterial gut microbiota due to prolonged antibiotic usage and the subsequent colonization with $C$. albicans interfere with the reassembly of the bacterial community structure, resulting in altered abundances of Bacteriodetes, Lactobacillaceae, Ruminococcaceae, and Lachnospiraceae [56]. Since the two different microbial communities (fungi and bacteria) mutually influence, we could also speculate that the reduced early life encounters with foodborne and environmental bacteria and fungi in urban areas of the globalized world could be a cause of the increased colonization with some major commensals, such as the pathobionts Candida and Escherichia. The alteration of the composition of the gut microbiota could also be mediated by mechanism of trained immunity [57] or by a reduced ability of the immune system to control its overgrowth due to lack of immune training, extending the hygiene hypothesis [58] from bacteria to yeasts [59].

\section{Conclusions}

Here, we observed an altered intestinal microbial community associated with ASDs, both at bacterial and fungal level not depending by the constipation status of autistic individuals but rather by the autistic disorder itself. However, due to the broad phenotypical variability of ASDs, an in-depth characterization of the genetic and phenotypical background in a larger cohort of ASD individuals would be necessary to comprehensively understand the role of the gut microbiota in ASDs pathophysiology and to further validate these findings. Our results therefore encourage new extensive, multicentric studies on the impact of the bacterial and fungal components of the gut microbiota in the gastrointestinal physiology and neuroplastic changes in ASDs, as well as the integration of such data with genetics, immunology, and metabolomics to further establish the relevance of the gut microbiota in the ASDs.

\section{Methods}

Study participants and samples' handling and collection We recruited 40 subjects with clinical diagnosis of autism (average age 11.1 \pm 6.8 ; sex, male:female, 31:9) and we compared them with 40 age and sex-matched neurotypical healthy subjects (average age 9.2 \pm 7.9 ; sex, male:female, 28:12). Autistic subjects with clinically evident inflammatory conditions were excluded. Constipation and inflammation (i.e., serum IgA, erythrocyte sedimentation rate, and faecal calprotectin levels) were also assessed. The autistic subjects were consecutively admitted to the Child Neuropsychiatry Unit of the University Hospital of Siena, and ASDs were diagnosed according with the Diagnostic and Statistical Manual of Mental Disorders, 5th Edition [1], and evaluated using Autism Diagnostic Observation Schedule and Autism Behaviour Checklist. Childhood Autism Rating Scale (CARS) scores [60] were calculated by an experienced child neuropsychiatrist. Average CARS values were $46.2 \pm 6.8$ (value range $32-57$ ); a fraction of $90 \%(36 / 40)$ were classified as severe ASDs (CARS value $>37$ ), with $10 \%(4 / 40)$ being moderately severe ASDs (CARS values from 30 to 36) (Additional file 12: Table S9). No specific comorbidities in the autistic cohort were present with the single exception of a coexisting celiac disease in two patients (5\%).

Constipation has been defined according to Rome III criteria [61]. Stool samples from enrolled subjects were collected, aliquoted as it is, and stored at $-80{ }^{\circ} \mathrm{C}$ until analysis. All subjects of this study were under a Mediterranean-based diet, and no antibiotics, probiotics, or prebiotics have been taken in the 3 months prior to the sample collection. None of the subjects were on anti-inflammatory or antioxidant drugs. The study was conducted after the approval by the Institutional Review Board of the Siena University Hospital (AOUS, Siena, Italy) and all written informed consents were obtained from either the parents or the legal tutors of the enrolled subjects, in compliance with national legislation and the Code of Ethical Principles for Medical Research Involving Human Subjects of the World Medical Association (Declaration of Helsinki).

\section{Faecal calprotectin assay}

Calprotectin determination was performed by using a polyclonal antibody in an enzyme-linked immunosorbent assay (Calprest, Eurospital, Trieste, Italy) according to the manufacturer's instructions. Calprotectin values $<50 \mu \mathrm{g} / \mathrm{g}$ per stool sample were considered normal.

\section{Pyrosequencing and data analysis}

Total DNA extraction from faecal samples $(250 \mathrm{mg}$, wet weight) was performed using the FastDNA ${ }^{\mathrm{mm}}$ SPIN Kit for Feces (MP Biomedicals, Santa Ana, CA, USA) 
following manufacturer's instructions. For each DNA sample, we amplified respectively the bacterial 16S rRNA genes using a primer set specific for V3-V5 hypervariable regions (F357: 5' -TCCTACGGGAGGCAGCAG-3' and R937: 5' -TGTGCGGGCCCCCGTCAATT-3') and the internal transcribed spacer (ITS) using a primer set specific for fungal ITS1 rDNA region (18SF: 5'-GTAAAAGTCGTAACAAGGTTTC-3' and 5.8S1R: 5'-GTTCAAAGAYT CGATGATTCAC-3') [62] containing adaptors, key sequence, and barcode sequences as described by the 454 Sequencing System Guidelines for Amplicon Experimental Design (Roche, Basel, Switzerland). The PCR products obtained were then purified, quantified, and pooled in equimolar way in a final amplicon library. The 454 pyrosequencing was carried out on the GS FLX+ system using the $\mathrm{XL}+$ chemistry following the manufacturer's recommendations (Roche, Basel, Switzerland). Raw 454 data were demultiplexed using the Roche's sff file software and submitted to the European Nucleotide Archive (ENA) with accession numbers PRJEB15418 and PRJEB15420. Sample accessions IDs and metadata are available in Additional file 12: Table S9. Reads were preprocessed using the MICCA pipeline (v. 0.1) (http://www.micca.org/) [63]. Operational taxonomic units (OTUs) were assigned by clustering the sequences with a threshold of $97 \%$ pairwise identity andv their representative sequences were classified using the RDP classifier version 2.7 on $16 \mathrm{~S}$ data and using the RDP classifier version 2.8 [64] against the UNITE fungal ITS database [65] on ITS1 data. Templateguided multiple sequence alignment (MSA) was performed using PyNAST [66] (v. 0.1) against the multiple alignment of the Greengenes [67] database (release 13_05) filtered at $97 \%$ similarity for bacterial sequences and through de novo MSA using T-Coffee [68] for fungal sequences. Fungal taxonomy assignments were then manually curated using BLASTn against the GenBank's database for accuracy. High-quality fungal sequences have been also manually filtered out for sequences belonging to Agaricomycetes (unlikely to be residents of the human gut due to their ecology [69]). The phylogenetic tree was inferred using micca-phylogeny [70]. Sampling heterogeneity was reduced by rarefaction. Alpha (within-sample richness) and beta-diversity (between-sample dissimilarity) estimates were computed using the phyloseq $\mathrm{R}$ package [71]. PERMANOVA test was performed using the adonis() function in the R package vegan with 999 permutations. Permutations have been constrained within age groups (corresponding to $0-2,3-10,11-17$, and $>18$ years old) or gender to evaluate possible biases related to the unequal age and gender distributions among subjects using the "strata" argument within the adonis() function. Two-sided, unpaired Welch $t$ statistics were computed using the function $\mathrm{mt}($ ) in the phyloseq library and the $p$ values were adjusted for multiple comparisons controlling the family- wise type I error rate (minP procedure) [72]. Spearman's correlation tests were computed using the psych $\mathrm{R}$ package [73]. Linear discriminant effect size (LEfSe) analysis was performed to find features (taxa) differentially represented between autistic and neurotypical subjects. LEfSe combines Kruskal-Wallis test or pairwise Wilcoxon ranksum test with linear discriminant analysis (LDA). It ranks features by effect size, which put features that explain most of the biological difference at top. LEfSe analysis was performed under the following conditions: $\alpha$ value for the statistical test equal to 0.01 and threshold on the logarithmic LDA score for discriminative features equal to 2.0 [29]. All statistical analyses were performed using $\mathrm{R}$ [74], and $p$ values were FDR corrected [75].

\section{Additional files}

Additional file 1: Table S1. Statistical comparisons (Wilcoxon rank-sum test) of clinical data among autistic (AD) and neurotypical (NT) subjects both constipated (C) and non-constipated (NC). (PDF 85 kb)

Additional file 2: Table S2. Permutational multivariate analysis of variance (PERMANOVA) tests of the bacterial gut microbiota on the unweighted and weighted UniFrac distances and the Bray-Curtis dissimilarity according to individuals' health status and constipation. (PDF $160 \mathrm{~kb}$ )

Additional file 3: Table S3. Permutational multivariate analysis of variance (PERMANOVA) tests of the bacterial and fungal gut microbiota on the unweighted and weighted UniFrac distances and the Bray-Curtis dissimilarity according to the severity of the autistic phenotype. (PDF $11 \mathrm{~kb}$ )

Additional file 4: Figure S1. Relative abundances at the genus level of the bacterial gut microbiota of autistic (AD) and neurotypical (NT) subjects both constipated (C) and non-constipated (NC). (PDF $1830 \mathrm{~kb}$ )

Additional file 5: Table S4. Mean relative abundance (\%) \pm standard deviation (SD) of bacterial taxa at genus levels in autistic (AD) subjects and neurotypical (NT) controls subjects both constipated (C) and non-constipated (NC). (PDF 303 kb)

Additional file 6: Spearman's correlation analysis among the most abundant bacterial genera and the constipation status of the subjects of the study cohort. (PDF $154 \mathrm{~kb}$ )

Additional file 7: Table S6. Permutational multivariate analysis of variance (PERMANOVA) tests of the fungal gut microbiota on the unweighted and weighted UniFrac distances and the Bray-Curtis dissimilarity according to individuals' health status and constipation. (PDF 159 kb)

Additional file 8: Figure S2. Relative abundances at the genus level of the fungal gut microbiota of autistic (AD) and neurotypical (NT) subjects both constipated (C) and non-constipated (NC). (PDF 346 kb)

Additional file 9: Table S7. Mean relative abundance (\%) \pm standard deviation (SD) of fungal taxa at genus levels in autistic (AD) subjects and neurotypical (NT) controls subjects both constipated (C) and nonconstipated (NC). (PDF $199 \mathrm{~kb}$ )

Additional file 10: Figure S3. Candida relative abundance in autistic (AD) and neurotypical (NT) subjects. Candida relative abundances are reported as mean \pm standard error. (PDF $19 \mathrm{~kb}$ )

Additional file 11: Table S8. Spearman's correlation analysis among the most abundant bacterial genera and fungal genera in autistic and neurotypical subjects. (PDF $253 \mathrm{~kb}$ )

Additional file 12: Table S9. Correspondences among deposited metagenomic data and samples. (XLS $70 \mathrm{~kb}$ ) 


\section{Abbreviations}

AD: Autistic subjects; ASDs: Autism spectrum disorders; CARS: Childhood Autism Rating Scale; CNS: Central nervous system; ESR: Erythrocyte sedimentation rate; FDR: False discovery rate; Gl: Gastrointestinal; IBD: Inflammatory bowel disease; ITS: Internal transcribed spacer; NT: Neurotypical subjects; OTU: Operational taxonomic unit; PCoA: Principal coordinates analysis; PERMANOVA: Permutational multivariate analysis of variance; SCFAs: Short chain fatty acids

\section{Acknowledgements}

The authors would like to thank Maddalena Sordo, Irene Stefanini, Massimo Pindo, and the lab staff of the Sequencing Platform from Fondazione Edmund Mach for technical support and the helpful discussions. We thank Unifarm S.p.A. for financial support to FS. We thank Cesare Furlanello and Paolo Zanini for the insightful discussions on the connection between diet, the globalized world, microbiota, and autism. All the authors are grateful to the autistic subjects and their families.

\section{Funding}

This work was supported by the "Accordo di Programma Integrato MetaFoodLabs" and funded by the research office of the Provincia Autonoma di Trento (Italy) (PAT Prot. S116/2012/537723).

\section{Availability of data and materials}

Raw sequences are available in the European Nucleotide Archive (ENA) with accession numbers PRJEB15418 and PRJEB15420.

\section{Authors' contributions}

FS, CDF, DC, and AC conceived the study and designed the experiments. FS performed the experiments and wrote the manuscript. DR and SL analysed and collected the clinical data. FS analysed the metagenomic data. DA and CD supervised and contributed to data analysis. CDFe, JH, SL, DR, and $A C$ recruited subjects and collected specimens. $C D F e, J H, S L, D R$, and $A C$ provided further clinical patient information and reagents for the study. AC, DC, and CDF approved the final manuscript. All the authors critically reviewed and approved the manuscript.

\section{Competing interests}

The authors declare that they have no competing interests.

\section{Consent for publication}

Not applicable.

\section{Ethics approval and consent to participate}

The study was conducted after the approval by the Institutional Review Board of the Siena University Hospital (AOUS, Siena, Italy), and all written informed consents were obtained from either the parents or the legal tutors of the enrolled subjects, in compliance with national legislation and the Code of Ethical Principles for Medical Research Involving Human Subjects of the World Medical Association (Declaration of Helsinki).

\footnotetext{
Author details

${ }^{1}$ Computational Biology Research Unit, Research and Innovation Centre, Fondazione Edmund Mach, Via E. Mach 1, 38010 San Michele all' Adige, Italy. ${ }^{2}$ Centre for Integrative Biology, University of Trento, Via Sommarive 9, 38123 Trento, Italy. ${ }^{3}$ Department of Biology, University of Florence, Via Madonna del Piano 6, 50019 Sesto Fiorentino, Florence, Italy. ${ }^{4}$ Neonatal Intensive Care Unit, Siena University Hospital AOUS, Viale Bracci 16, 53100 Siena, Italy. ${ }^{5} \mathrm{C}$ ild Neuropsychiatry Unit, Siena University Hospital AOUS, Viale Bracci 16, 53100 Siena, Italy. ${ }^{6}$ Azienda Unità Sanitaria Locale Umbria 2, Via D. Bramante 37, 05100 Terni, Italy. ${ }^{7}$ Department of Experimental and Clinical Biomedical Sciences, Gastroenterology Unit, University of Florence, Viale Morgagni 40, 50139 Florence, Italy. Institute of Agriculture Biology and Biotechnology, National Research Council (CNR), Via Moruzzi 1, 56124 Pisa, Italy.
}

Received: 20 September 2016 Accepted: 7 February 2017

Published online: 22 February 2017

\section{References}

1. Association AP. Diagnostic and statistical manual of mental disorders $\left(\right.$ DSM-5 $\left.{ }^{\circledR}\right)$ : American Psychiatric Pub; 2013.

2. Kim YS, Leventhal BL. Genetic epidemiology and insights into interactive genetic and environmental effects in autism spectrum disorders. Biol Psychiatry. 2015;77(1):66-74.

3. Colvert E, Tick B, McEwen F, Stewart C, Curran SR, Woodhouse E, Gillan N, Hallett V, Lietz S, Garnett T, et al. Heritability of autism spectrum disorder in a UK population-based twin sample. JAMA Psychiatry. 2015;72(5):415-23.

4. McElhanon BO, McCracken C, Karpen S, Sharp WG. Gastrointestinal symptoms in autism spectrum disorder: a meta-analysis. Pediatrics. 2014;133(5):872-83.

5. Hsiao EY. Gastrointestinal issues in autism spectrum disorder. Harv Rev Psychiatry. 2014;22(2):104-11.

6. Furuta GT, Williams K, Kooros K, Kaul A, Panzer R, Coury DL, Fuchs G. Management of constipation in children and adolescents with autism spectrum disorders. Pediatrics. 2012;130 Suppl 2:S98-105.

7. Gorrindo P, Williams KC, Lee EB, Walker LS, McGrew SG, Levitt P. Gastrointestinal dysfunction in autism: parental report, clinical evaluation, and associated factors. Autism Res. 2012;5(2):101-8.

8. Mayer EA, Padua D, Tillisch K. Altered brain-gut axis in autism: comorbidity or causative mechanisms? BioEssays. 2014;36(10):933-9.

9. Sampson TR, Mazmanian SK. Control of brain development, function, and behavior by the microbiome. Cell Host Microbe. 2015;17(5):565-76.

10. Barajon I, Serrao G, Arnaboldi F, Opizzi E, Ripamonti G, Balsari A, Rumio C. Toll-like receptors 3, 4, and 7 are expressed in the enteric nervous system and dorsal root ganglia. J Histochem Cytochem. 2009;57(11):1013-23.

11. Brun P, Giron MC, Qesari M, Porzionato A, Caputi V, Zoppellaro C, Banzato S, Grillo AR, Spagnol L, De Caro R, et al. Toll-like receptor 2 regulates intestinal inflammation by controlling integrity of the enteric nervous system. Gastroenterology. 2013;145(6):1323-33.

12. Sudo N. Role of microbiome in regulating the HPA axis and its relevance to allergy. Chem Immunol Allergy. 2012;98:163-75.

13. De Vadder F, Kovatcheva-Datchary P, Goncalves D, Vinera J, Zitoun C, Duchampt A, Backhed F, Mithieux G. Microbiota-generated metabolites promote metabolic benefits via gut-brain neural circuits. Cell. 2014;156(1-2):84-96.

14. Ray K. Gut microbiota: microbial metabolites feed into the gut-brain-gut circuit during host metabolism. Nat Rev Gastroenterol Hepatol. 2014;11(2):76.

15. Kamada N, Seo SU, Chen GY, Nunez G. Role of the gut microbiota in immunity and inflammatory disease. Nat Rev Immunol. 2013;13(5):321-35.

16. Berer K, Krishnamoorthy G. Commensal gut flora and brain autoimmunity: a love or hate affair? Acta Neuropathol. 2012;123(5):639-51.

17. Stolp HB, Dziegielewska KM, Ek CJ, Potter AM, Saunders NR. Long-term changes in blood-brain barrier permeability and white matter following prolonged systemic inflammation in early development in the rat. Eur J Neurosci. 2005;22(11):2805-16.

18. Onore CE, Nordahl CW, Young GS, Van de Water JA, Rogers SJ, Ashwood P. Levels of soluble platelet endothelial cell adhesion molecule-1 and P-selectin are decreased in children with autism spectrum disorder. Biol Psychiatry. 2012;72(12):1020-5.

19. Onore C, Careaga M, Ashwood P. The role of immune dysfunction in the pathophysiology of autism. Brain Behav Immun. 2012;26(3):383-92.

20. Theoharides TC, Asadi S, Patel AB. Focal brain inflammation and autism. J Neuroinflammation. 2013;10:46.

21. Stolp HB, Johansson PA, Habgood MD, Dziegielewska KM, Saunders NR, Ek CJ. Effects of neonatal systemic inflammation on blood-brain barrier permeability and behaviour in juvenile and adult rats. Cardiovasc Psychiatry Neurol. 2011;2011:469046.

22. Oever JT, Netea MG. The bacteriome-mycobiome interaction and antifungal host defense. Eur J Immunol. 2014;44(11):3182-91.

23. De Luca A, Montagnoli C, Zelante T, Bonifazi P, Bozza S, Moretti S, D'Angelo C, Vacca C, Boon L, Bistoni F, et al. Functional yet balanced reactivity to Candida albicans requires TRIF, MyD88, and IDO-dependent inhibition of Rorc. J Immunol. 2007;179(9):5999-6008.

24. Cheng SC, van de Veerdonk F, Smeekens S, Joosten LA, van der Meer JW, Kullberg BJ, Netea MG. Candida albicans dampens host defense by downregulating IL-17 production. J Immunol. 2010;185(4):2450-7. 
25. Bozza S, Fallarino F, Pitzurra L, Zelante T, Montagnoli C, Bellocchio S, Mosci P, Vacca C, Puccetti P, Romani L. A crucial role for tryptophan catabolism at the host/Candida albicans interface. J Immunol. 2005;174(5):2910-8.

26. Vandeputte D, Falony G, Vieira-Silva S, Tito RY, Joossens M, Raes J. Stool consistency is strongly associated with gut microbiota richness and composition, enterotypes and bacterial growth rates. Gut. 2016;65(1):57-62.

27. Zhu L, Liu W, Alkhouri R, Baker RD, Bard JE, Quigley EM, Baker SS. Structural changes in the gut microbiome of constipated patients. Physiol Genomics. 2014;46(18):679-86.

28. Kang DW, Park JG, Ilhan ZE, Wallstrom G, Labaer J, Adams JB, KrajmalnikBrown R. Reduced incidence of Prevotella and other fermenters in intestinal microflora of autistic children. PLoS One. 2013;8(7):e68322.

29. Segata N, Izard J, Waldron L, Gevers D, Miropolsky L, Garrett WS, Huttenhower C. Metagenomic biomarker discovery and explanation. Genome Biol. 2011;12(6):R60

30. Zoppi G, Cinquetti M, Luciano A, Benini A, Muner A, Bertazzoni Minelli E. The intestinal ecosystem in chronic functional constipation. Acta Paediatr. 1998;87(8):836-41.

31. Son JS, Zheng LJ, Rowehl LM, Tian X, Zhang Y, Zhu W, Litcher-Kelly L, Gadow KD, Gathungu G, Robertson CE, et al. Comparison of fecal microbiota in children with autism spectrum disorders and neurotypical siblings in the Simons simplex collection. PLoS One. 2015;10(10):e0137725.

32. Wang $\mathrm{Y}$, Kasper $\mathrm{LH}$. The role of microbiome in central nervous system disorders. Brain Behav Immun. 2014;38:1-12.

33. Strati F, Cavalieri D, Albanese D, De Felice C, Donati C, Hayek J, Jousson O, Leoncini S, Pindo M, Renzi D, et al. Altered gut microbiota in Rett syndrome. Microbiome. 2016;4(1):41

34. Frank DN, St Amand AL, Feldman RA, Boedeker EC, Harpaz N, Pace NR. Molecular-phylogenetic characterization of microbial community imbalances in human inflammatory bowel diseases. Proc Natl Acad Sci U S A. 2007;104(34):13780-5.

35. Turnbaugh PJ, Backhed F, Fulton L, Gordon J. Diet-induced obesity is linked to marked but reversible alterations in the mouse distal gut microbiome. Cell Host Microbe. 2008;3(4):213-23.

36. Williams BL, Hornig M, Buie T, Bauman ML, Cho Paik M, Wick I, Bennett A, Jabado O, Hirschberg DL, Lipkin WI. Impaired carbohydrate digestion and transport and mucosal dysbiosis in the intestines of children with autism and gastrointestinal disturbances. PLoS One. 2011;6(9):e24585.

37. Tomova A, Husarova V, Lakatosova S, Bakos J, Vlkova B, Babinska K, Ostatnikova D. Gastrointestinal microbiota in children with autism in Slovakia. Physiol Behav. 2015;138:179-87.

38. Hsiao EY, McBride SW, Hsien S, Sharon G, Hyde ER, McCue T, Codelli JA, Chow J, Reisman SE, Petrosino JF, et al. Microbiota modulate behavioral and physiological abnormalities associated with neurodevelopmental disorders. Cell. 2013;155(7):1451-63.

39. Finegold SM, Dowd SE, Gontcharova V, Liu C, Henley KE, Wolcott RD, Youn E, Summanen PH, Granpeesheh D, Dixon D, et al. Pyrosequencing study of fecal microflora of autistic and control children. Anaerobe. 2010;16(4):444-53.

40. Adams JB, Johansen L, Powell LD, Quig D, Rubin RA. Gastrointestinal flora and gastrointestinal status in children with autism-comparisons to typical children and correlation with autism severity. BMC Gastroenterol. 2011;11:22.

41. Morgan XC, Tickle TL, Sokol H, Gevers D, Devaney KL, Ward DV, Reyes JA, Shah SA, LeLeiko N, Snapper SB, et al. Dysfunction of the intestinal microbiome in inflammatory bowel disease and treatment. Genome Biol. 2012;13(9):R79

42. Arthur JC, Gharaibeh RZ, Mühlbauer M, Perez-Chanona E, Uronis JM, McCafferty J, Fodor AA, Jobin C. Microbial genomic analysis reveals the essential role of inflammation in bacteria-induced colorectal cancer. Nat Commun. 2014;5.

43. Frye RE, Rose S, Slattery J, MacFabe DF. Gastrointestinal dysfunction in autism spectrum disorder: the role of the mitochondria and the enteric microbiome. Microb Ecol Health Dis. 2015;26:27458.

44. Stiles BG, Pradhan K, Fleming JM, Samy RP, Barth H, Popoff MR. Clostridium and bacillus binary enterotoxins: bad for the bowels, and eukaryotic being. Toxins. 2014;6(9):2626-56.

45. Matsuda H, Fujiyama Y, Andoh A, Ushijima T, Kajinami T, Bamba T. Characterization of antibody responses against rectal mucosa-associated bacterial flora in patients with ulcerative colitis. J Gastroenterol Hepatol. 2000;15(1):61-8.

46. Woting A, Pfeiffer N, Loh G, Klaus S, Blaut M. Clostridium ramosum promotes high-fat diet-induced obesity in gnotobiotic mouse models. MBio. 2014;5(5):e01530-01514.
47. Atarashi K, Tanoue T, Oshima K, Suda W, Nagano Y, Nishikawa H, Fukuda S, Saito T, Narushima S, Hase K. Treg induction by a rationally selected mixture of Clostridia strains from the human microbiota. Nature. 2013;500(7461):232-6.

48. Cortelazzo A, De Felice C, Guerranti R, Signorini C, Leoncini S, Zollo G, Leoncini R, Timperio AM, Zolla L, Ciccoli L, Hayek J. Expression and oxidative modifications of plasma proteins in autism spectrum disorders: Interplay between inflammatory response and lipid peroxidation. Proteomics Clin Appl. 2016;10(11):1103-12.

49. Rizzetto L, De Filippo C, Cavalieri D. Richness and diversity of mammalian fungal communities shape innate and adaptive immunity in health and disease. Eur J Immunol. 2014:44(11):3166-81.

50. Scanlan PD, Marchesi JR. Micro-eukaryotic diversity of the human distal gut microbiota: qualitative assessment using culture-dependent and -independent analysis of faeces. ISME J. 2008;2(12):1183-93.

51. Strati F, Di Paola M, Stefanini I, Albanese D, Rizzetto L, Lionetti P, Calabro A, Jousson O, Donati C, Cavalieri D, et al. Age and gender affect the composition of fungal population of the human gastrointestinal tract. Front Microbiol. 2016;7:1227

52. Li Q, Wang C, Tang C, He Q, Li N, Li J. Dysbiosis of gut fungal microbiota is associated with mucosal inflammation in Crohn's disease. J Clin Gastroenterol. 2014:48(6):513.

53. Luan C, Xie L, Yang X, Miao H, Lv N, Zhang R, Xiao X, Hu Y, Liu Y, Wu N, et al. Dysbiosis of fungal microbiota in the intestinal mucosa of patients with colorectal adenomas. Sci Rep. 2015;5:7980.

54. Iliev ID, Funari VA, Taylor KD, Nguyen Q, Reyes CN, Strom SP, Brown J, Becker CA, Fleshner PR, Dubinsky M, et al. Interactions between commensal fungi and the C-type lectin receptor Dectin-1 influence colitis. Science. 2012;336(6086):1314-7.

55. Zelante T, lannitti RG, Cunha C, De Luca A, Giovannini G, Pieraccini G, Zecchi R, D'Angelo C, Massi-Benedetti C, Fallarino F, et al. Tryptophan catabolites from microbiota engage aryl hydrocarbon receptor and balance mucosal reactivity via interleukin-22. Immunity. 2013;39(2):372-85.

56. Erb Downward JR, Falkowski NR, Mason KL, Muraglia R, Huffnagle GB. Modulation of post-antibiotic bacterial community reassembly and host response by Candida albicans. Sci Rep. 2013;3:2191.

57. Rizzetto L, Ifrim DC, Moretti S, Tocci N, Cheng SC, Quintin J, Renga G, Oikonomou V, De Filippo C, Weil T, et al. Fungal chitin induces trained immunity in human monocytes during cross-talk of the host with Saccharomyces cerevisiae. J Biol Chem. 2016;291(15):7961-72.

58. Lowry CA, Smith DG, Siebler PH, Schmidt D, Stamper CE, Hassell Jr JE, Yamashita PS, Fox JH, Reber SO, Brenner LA, et al. The microbiota, immunoregulation, and mental health: implications for public health. Curr Environ Health Rep. 2016;3(3):270-86.

59. Wander K, O'Connor K, Shell-Duncan B. Expanding the hygiene hypothesis: early exposure to infectious agents predicts delayed-type hypersensitivity to Candida among children in Kilimanjaro. PLoS One. 2012;7(5):e37406.

60. Schopler E, Reichler RJ, DeVellis RF, Daly K. Toward objective classification of childhood autism: Childhood Autism Rating Scale (CARS). J Autism Dev Disord. 1980;10(1):91-103.

61. Longstreth GF, Thompson WG, Chey WD, Houghton LA, Mearin F, Spiller RC. Functional bowel disorders. Gastroenterology. 2006;130(5):1480-91.

62. Findley K, Oh J, Yang J, Conlan S, Deming C, Meyer JA, Schoenfeld D, Nomicos E, Park M, Program NIHISCCS, et al. Topographic diversity of fungal and bacterial communities in human skin. Nature. 2013;498(7454):367-70.

63. Albanese D, Fontana P, De Filippo C, Cavalieri D, Donati C. MICCA: a complete and accurate software for taxonomic profiling of metagenomic data. Sci Rep. 2015;5:9743.

64. Wang Q, Garrity GM, Tiedje JM, Cole JR. Naive Bayesian classifier for rapid assignment of rRNA sequences into the new bacterial taxonomy. Appl Environ Microbiol. 2007;73(16):5261-7.

65. Koljalg U, Nilsson RH, Abarenkov K, Tedersoo L, Taylor AF, Bahram M, Bates ST, Bruns TD, Bengtsson-Palme J, Callaghan TM, et al. Towards a unified paradigm for sequence-based identification of fungi. Mol Ecol. 2013;22(21):5271-7.

66. Caporaso JG, Bittinger K, Bushman FD, DeSantis TZ, Andersen GL, Knight R. PyNAST: a flexible tool for aligning sequences to a template alignment. Bioinformatics. 2010;26(2):266-7.

67. DeSantis TZ, Hugenholtz P, Larsen N, Rojas M, Brodie EL, Keller K, Huber T, Dalevi D, Hu P, Andersen GL. Greengenes, a chimera-checked 16S rRNA gene database and workbench compatible with ARB. Appl Environ Microbiol. 2006;72(7):5069-72. 
68. Notredame C, Higgins DG, Heringa J. T-Coffee: a novel method for fast and accurate multiple sequence alignment. J Mol Biol. 2000;302(1):205-17.

69. Hibbett DS. A phylogenetic overview of the Agaricomycotina. Mycologia. 2006;98(6):917-25.

70. Price MN, Dehal PS, Arkin AP. FastTree 2-approximately maximumlikelihood trees for large alignments. PLoS One. 2010;5(3):e9490.

71. McMurdie PJ, Holmes S. phyloseq: an R package for reproducible interactive analysis and graphics of microbiome census data. PLoS One. 2013;8(4):e61217.

72. Westfall PH, Young SS. Resampling-based multiple testing: examples and methods for p-value adjustment, vol. 279. New York: Wiley; 1993.

73. Revelle W. psych: procedures for psychological, psychometric, and personality research, R package, vol. 10. 13th ed. Evanston: Northwestern University; 2013

74. Team RC. R: a language and environment for statistical computing. $R$ Foundation for Statistical Computing, Vienna, Austria, 2012. In.: ISBN 3-900051-07-0; 2014

75. Benjamini Y, Hochberg Y. Controlling the False Discovery Rate: A Practical and Powerful Approach to Multiple Testing. Journal of the Royal Statistical Society. Series B (Methodological). 1995;57(1):289-300.

Submit your next manuscript to BioMed Central and we will help you at every step:

- We accept pre-submission inquiries

- Our selector tool helps you to find the most relevant journal

- We provide round the clock customer support

- Convenient online submission

- Thorough peer review

- Inclusion in PubMed and all major indexing services

- Maximum visibility for your research

Submit your manuscript at www.biomedcentral.com/submit
Biomed Central 